\title{
Femtosecond Micromachining of Ceramic Fibers for Electric Contact Soldering
}

\author{
Domas PAIPULAS ${ }^{* 1}$, Aleksandr ALESENKOV ${ }^{* 1}$, Dalia KAŠKELYTË ${ }^{* 1}$, Gediminas CHAŽEVSKIS ${ }^{* 1}$, Paulius RAGULIS ${ }^{* 2}$, \\ Žilvinas KANCLERIS ${ }^{* 2}$, Valdas SIRUTKAITIS ${ }^{* 1}$, \\ ${ }^{* 1}$ Vilnius University, Physics Faculty, Department of Quantum Electronics, Laser Research Center, \\ Saulètekio av. 10, LT-10223, Vilnius, Lithuania \\ *2 Center for Physical Sciences and Technology, A. Goštauto 11, LT-01108, Vilnius, Lithuania
}

E-mail: domas.paipulas@ff.vu.lt

\begin{abstract}
In this report we demonstrate that using femtosecond laser pulses it is possible to create micronotches and micro-wells in conductive titanium dioxide (Magnéli phase) ceramic. Fabricated microcavities were later used as support points for wire soldering in order to integrate ceramic fiber into electronic measuring circuit as a mechanical wear sensor. We show that with optimal cutting parameters laser-processed areas retain good conduction properties and have no impact on electric contact quality. Using galvanometric scanners together with high repetition rate femtosecond laser pulses it is possible to form such microcavities in times suitable for industrial needs.
\end{abstract}

DOI: 10.2961/jlmn.2013.03.0020

Keywords: Femtosecond micromachining, galvanoscanners, titanium dioxide, Magnéli phase, ceramic processing.

\section{Introduction}

Use of femtosecond laser pulses for micromachining purposes was demonstrated to be very successful in wide range of materials: transparent dielectrics, semiconductors, various metals and etc. [1-3], showing great potential and the versatility of the technique. Femtosecond laser ablation, because of the reduced heat-affected zone, where melting and solidification can occur, allows the micromachining and surface patterning of materials with minimal mechanical and thermal deformation, and with micrometer precision [4-5]. Also, a femtosecond laser technology has already become mature enough to be accessible for industry, which will not hesitate to unleash its full potential and provide us with new types of devices and solutions that were inspired or provided with femtosecond micromachining.

The development of advanced devices or sensors often requires unique engineering solutions that could be solved with laser micromachining. In this report we present the study of one of them. In order to create a sensor for monitoring the health of metallic mould installed in some inaccessible or remote area, conductive ceramic fiber has to be integrated into pre-cut groove on the surface of the mould [6]. Later, the resistance of the fiber has to be constantly monitored to access data about the wear level of the mould. In order to monitor resistance of the fiber, DC (direct current) circuit has to be formed and electric wires have to be attached to the ends of the ceramic fiber. Such procedure is not straightforward: fiber has to be prepared in a special way for sufficient and secure wire-to-fiber contact after the soldering. This preparation consists of creating microwells or notches in the fiber for precise wire positioning and reliable contact. In this process one must also prevent the change of conductive properties or thermal and mechanical degradation of the ceramics after microfabrication. Me- chanical cutting of the fiber lacks precision and could produce fiber fractures in brittle material, thus it is not reliable in this case. The ablation with nanosecond laser pulses is known to produce high thermal stress on the ceramic [7] that can influence ceramic resistivity and its use in our case is doubtful. We demonstrate that successful micromachining of titanium dioxide without the change of its properties could be achieved with ultrafast laser pulses.

\section{Experimental}

\subsection{Laser micromachining setup}

For micromachining task we used $\mathrm{Yb}: \mathrm{KGW}$ femtosecond laser (Pharos, "Light Conversion Ltd."), with 300 fs pulse duration at $1030 \mathrm{~nm}$ wavelength and scalable repetition rate in range of $25 \mathrm{kHz}-200 \mathrm{kHz}$. The maximum average laser power was $6 \mathrm{~W}$, however during micromachining a power was reduced with the help of optical attenuation system. Several experiments were performed using second harmonics (at $515 \mathrm{~nm}$ ).

We used micromachining approach based on galvanometric scanners. Computer controlled scanners (Intelscan10, "Scanlab") were used to deflect a laser beam to focusing optics, which, afterwards, focused laser beam on the target. Two different focusing configurations where used: f-theta lens and microscope objective used in conjunction with 4-f relay system. Both setups are shown in Fig.1. The most direct method to exploit micromachining using scanners is with f-theta lens. This lens acts as focusing optical element and is capable to produce aberration-free spot at wide working field. As focal length of such lens is rather long (in our experiment $-100 \mathrm{~mm}$ ), it is possible to achieve high linear beam scanning speeds on the focal plane $(>1 \mathrm{~m} / \mathrm{s})$. However, longer focal length results in the greater spot size which could be a drawback in some applications. Also, 


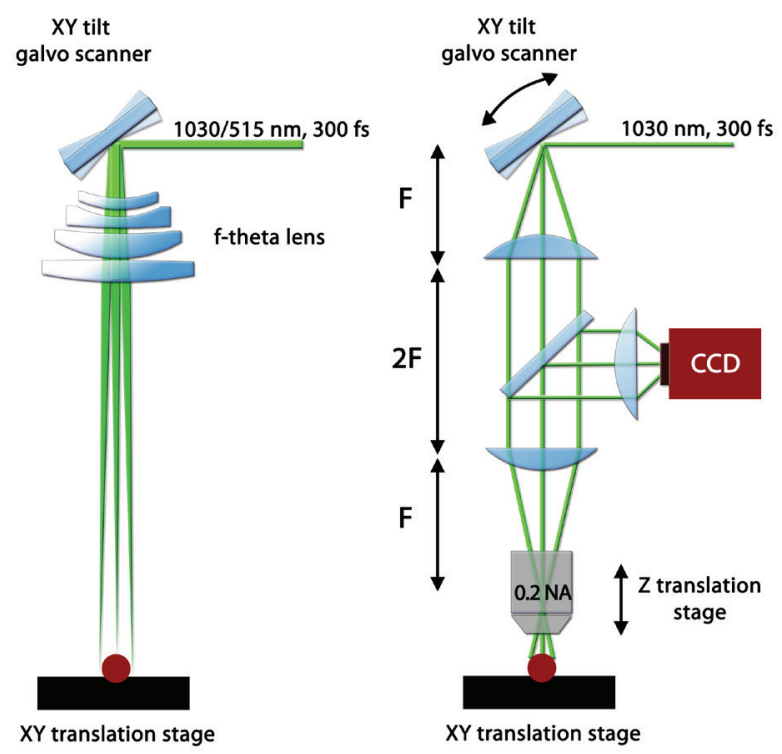

Fig. 1 (left) Sketch depicting micromachining using galvanometric scanners with: (left) f-theta type lens, (right) using conventional microscope objective and 4-f relay optical system.

installing visualization system for real time monitoring of processed area is rather complicated (but possible) in ftheta type systems. Visualization system is essential when precise positioning of the beam on the sample is necessary. This problem was solved in the second configuration. 4- $f$ relay system, consisting of two lenses (focal length $250 \mathrm{~mm}$, each), imaged scanners plane 1-to-1 onto entrance pupil of microscope objective (focal length $-25 \mathrm{~mm}$ and numerical aperture (NA) - 0.2), which focused laser beam onto the sample. In this configuration, beam spot size is determined by the focusing power of objective; however, scanners working area become much smaller (approx. 300 $\mu \mathrm{m})$ and focused spot is prone to aberrations at the field extremes. Nevertheless, in this configuration it is easy to install beam splitter and use it for visualization purposes. For precise positioning, sample was additionally mounted on XY translation stages ("Aerotech").

The conductive ceramic samples used in these experiments where prepared in Bath University and consisted of titanium dioxide at Magnéli phase $\left(\mathrm{Ti}_{\mathrm{n}} \mathrm{O}_{2 \mathrm{n}-1}\right.$, where $3<\mathrm{n}<10$ ) [8]. Ceramics were pressed in the fiber form from ball milled $\mathrm{TiO}_{2}$ powder and then sintered at high temperatures. Samples were $260 \mu \mathrm{m}$ in diameter. Fig. 2 shows SEM image of cross-section of typical ceramic fiber.

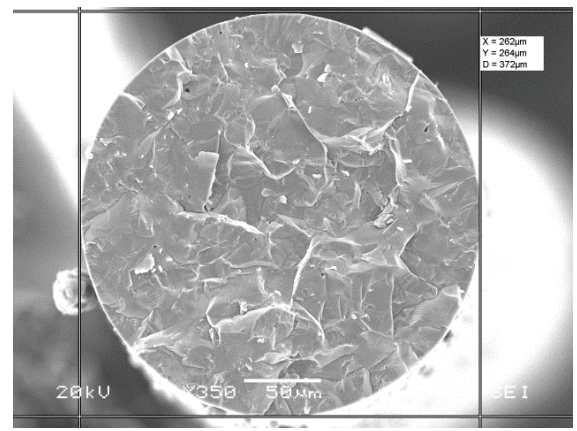

Fig. 2 SEM image of cross-section of titanium dioxide fiber.

\section{Micromachining of the ceramics}

Our main micromachining objective was to create approx. $130 \mu \mathrm{m}$ wide microcavities featuring $100 \mu \mathrm{m}$ depths to conceal $60 \mu \mathrm{m}$ silver wire. The most straightforward way to create up to $100 \mu \mathrm{m}$ depth trench in the ceramic fiber is to create a cut (notch) across the entire fiber diameter. As precise fiber positioning is not necessary in this case, micromachining is best realized with f-theta type system. Other possible variation is to form microwell directly on the fiber. For this task, fiber positioning is essential, thus it was carried out with 4- $f$ relay system.

\subsection{Notch fabrication}

In order to fabricate sufficient width notches, some patterning scheme is required. We used a spiral machining algorithm which is shown in Fig.3. In spiral machining algorithm the laser beam is scanned perpendicularly to the fiber (polarization was parallel to scanning direction) ablating one single line on fiber, while next lines are shifted by slight separation $(d)$ simultaneously from both sides. Number of lines in "the spiral" determines the width of the notch. To form the required depth of the notch, spiral cutting algorithm has to be repeated several times. Symmetrical cutting produces symmetrical walls of the notch. When conventional raster algorithm is used, one wall always tends to be asymmetric due to reattachment of the debris. This simple algorithm was demonstrated to be the best for microgroove formation on steel samples [9].

Typical notch made on the fiber with spiral algorithm is shown in Fig.4. Machining parameters were as follows:

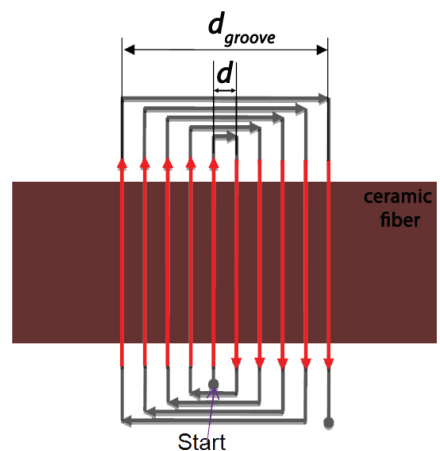

Fig. 3 Sketch of the spiral patterning algorithm when ablation starts from the central part of the notch. Sufficient depth of the notch is achieved by continuous repetition of this algorithm.

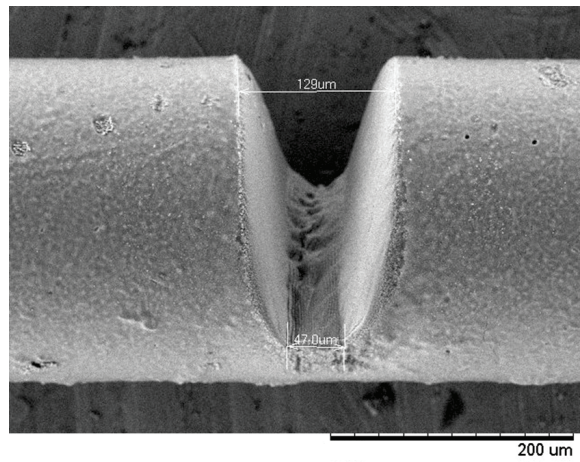

Fig. 4 Micronotch machined on ceramic fiber with spiral algorithm. 


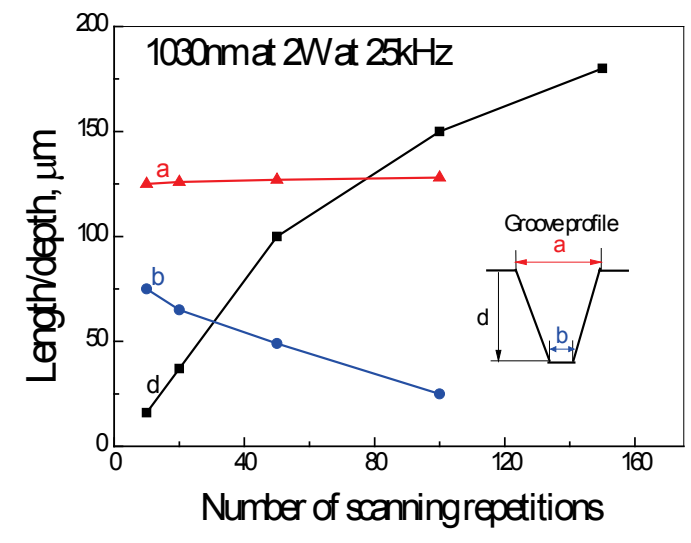

Fig. 5 Notch parameter dependence on spiral algorithm repetition number. Algorithm parameters: 24 lines with $5 \mu \mathrm{m}$ separation.

laser power $-2 \mathrm{~W}$, repetition rate $-25 \mathrm{kHz}$, linear scanning speed $-1 \mathrm{~m} / \mathrm{s}$, wavelength $-1030 \mathrm{~nm}$. Spiral algorithm consisted of 24 lines made with $5 \mu \mathrm{m}$ separation $(d)$, and the algorithm was repeated 50 times. This produced 100 $\mu \mathrm{m}$ depth notch having width of $\sim 130 \mu \mathrm{m}$ in less than $5 \mathrm{~s}$. Notch depth can be easily scaled by changing just algorithm repetition number, while retaining the same notch width as shown in Fig.5. As one can see from the figures, the groove entry width is always bigger than the bottom width. In our experiments we achieved $\sim 20^{\circ}$ steepness of the groove.

Similar notches were also created with $515 \mathrm{~nm}$ wavelength with all other cutting and algorithm parameters the same, but no apparent difference was observed.

\subsection{Microwell formation}

The circular algorithm was used for microwell formation where cutting was performed in concentric circles starting from the center with constantly increasing radius $(d)$ (Fig. 6). As in the case of notch fabrication, the number of circles in the algorithm determines the diameter of the well, while well depth is increased by additional repetition of the algorithm. Fig. 7 shows the examples of microwells made with following parameters: average laser power $-50 \mathrm{~mW}$ at $25 \mathrm{kHz}$, linear scanning speed $-1 \mathrm{~mm} / \mathrm{s}$, wavelength $1030 \mathrm{~nm}$. Algorithm consisted of 16 circles made with

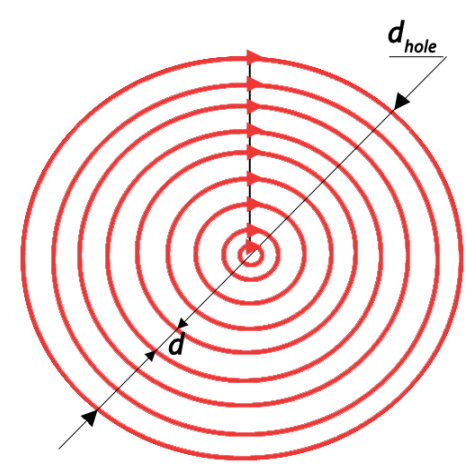

Fig. 6 Microwell fabrication algorithm, when cutting is started from the center towards the outside in circles with constantly increasing radius.
$5 \mu \mathrm{m}$ separation $(d)$, and all algorithm was repeated 50 times. Focal point of the objective was positioned directly on the fiber surface. This produced $80 \mu \mathrm{m}$ diameter microwell with $160 \mu \mathrm{m}$ depth. Micromachined fiber was later cleaned in ultrasonic bath to remove excessive debris.

One microwell was formed in approx. $5 \mathrm{~min}$. The increase of manufacturing time is fated by a linear scanning speed which becomes lower due to higher focusing. If precise positioning could be integrated in f-theta system, drilling time of equivalent microwell could be decreased by three orders.

As can be seen from the pictures, the overall quality of microwell is very good. At the well boundary small amount of reattached debris or (possible?) remelted material can be observed but this layer does not exceed $6 \mu \mathrm{m}$ and do not impact on conductive properties of the ceramics. Microwell
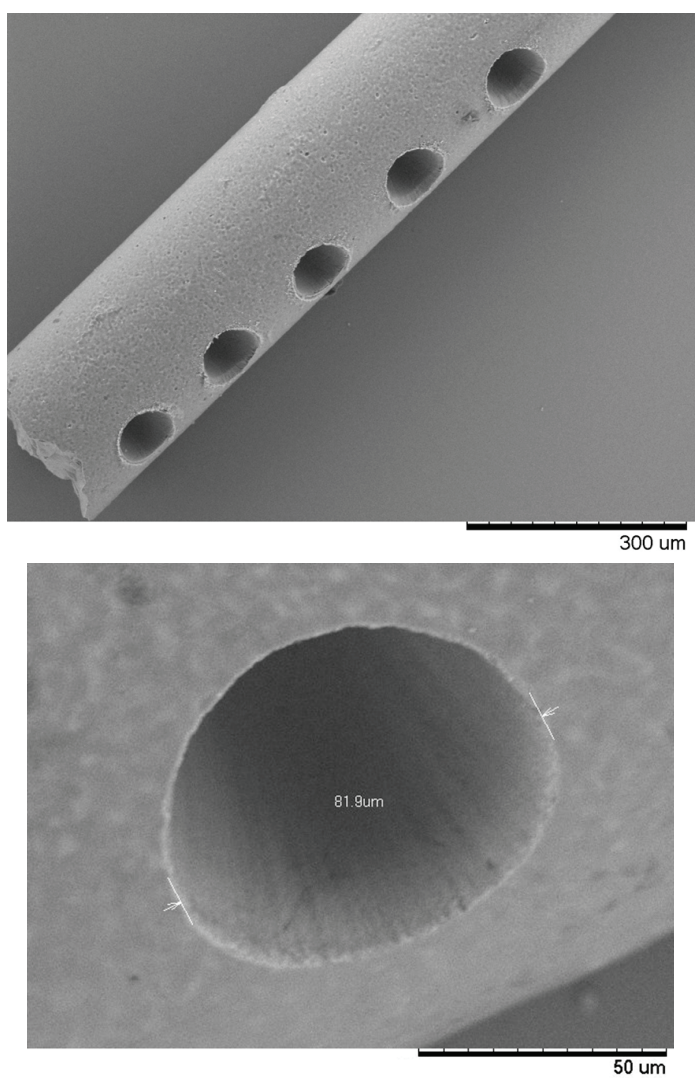

Fig. 7 Microwells machined in ceramic fibers. Well's depth is $160 \mu \mathrm{m}$.

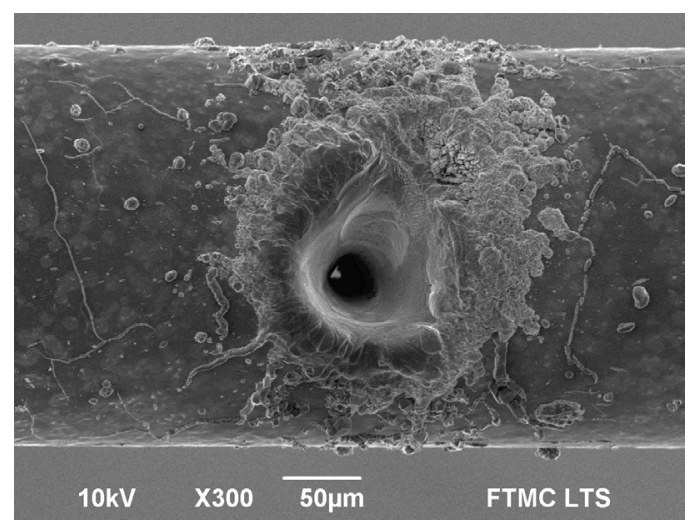

Fig. 8 Remelted slag, formed on the boundary of ablated well when fabricated with too high pulse power and repetition rate. 
bottom roughness was $<4 \mu \mathrm{m}$ (RMS).

The absence of the slag is the key result in this experiment. Such slag is present when material is micromachined with nanosecond or picosecond duration laser pulses. Remelted material experiences strong thermal stress and could have different physical properties (for example, loss of conductance) and is undesirable in our application. Similar slag could also appear when processing with femtosecond laser pulses. If laser repetition rate is set too high, thermal accumulation may develop in the material resulting in the sufficient increase of the temperature and consequent melting. For example, a microwell, drilled with $1.6 \mathrm{~W}$ laser power at $100 \mathrm{kHz}$ pulse repetition rate (at $515 \mathrm{~nm}$ wavelength) is shown in Fig. 8. The quality of this microcavity is much worse than shown in previous examples, where visible slag of remelted material is clearly present. It was not possible to create desirable electric contact in this case, as infinite resistance was observed between the contacts. This demonstrates that the remelted ceramic loses its conductive properties and this should be avoided.

\section{Resistivity analysis}

\subsection{Contact preparation and measurement technique}

When performing the investigation of electrical properties of fragile ceramic fiber in a DC electric field, the metal wires are usually attached to the ends of it, providing the connection of the fiber to the measuring circuit. In order to do that various metal pastes are used with follow-up annealing at high temperature to attain the mechanical strength of the contact and improve its electrical properties. The fiber with wires processed in this way is shown schematically in Fig. 9a. Using such technique a metal paste usually covers side surfaces of the fiber, which could be undesirable due to sensor's design; also, mechanical strength of such contact is poor, because pasting is performed on the flat surface. Wishing to overcome both shortcomings, micromachined wells (ref. to Fig. 9b) and notches (ref to Fig. 9c) in the fiber were used for fixing of contact wire. Such contact fixture point prevented the spread of the metal paste and localized a contact area. Mechanical strength of the connection was also improved due to increased pasting surface area. Comparing measurement results further in this paper we shall refer to letters $\mathrm{A}, \mathrm{B}$, and $\mathrm{C}$ when considering particular type of the wire connection as shown in the subfigures of Fig. 9.
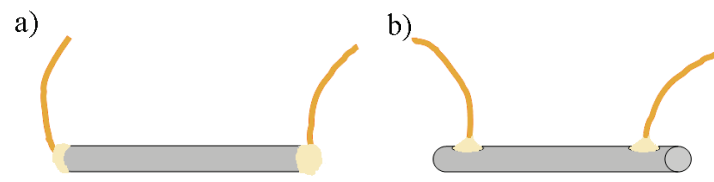

c)

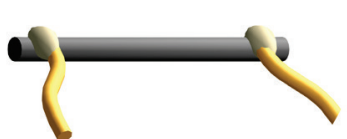

Fig. 9 Schematic view of the fibers with attached wires: (a) wires attached to the sides of the fiber, (b) wires fixed in the wells, (c) wires fixed in the notches. Capital letters further in the text correspond to the particular type of the connection depicted in this figure.
Liquid aluminum (Al) paste was used for contact preparation and wiring. Paste was spread on the end of the fiber (or into the cavity drilled in it) together with Ag wire at a room temperature. When one of the contacts was ready, the sample was heated at roughly $100^{\circ} \mathrm{C}$ for $10 \mathrm{~min}$. The $\mathrm{Al}$ paste becomes hard enough to perform the same procedure with the other contact. The samples with contacts were annealed in $\mathrm{N}_{2}$ atmosphere at $700^{\circ} \mathrm{C}$ for $30 \mathrm{~min}$. They were slowly inserted into high temperature zone of the furnace (10 min), were left in it for $10 \mathrm{~min}$, and were slowly drawn out $(10 \mathrm{~min})$ reaching room temperature. At the moment such contact processing and wire bonding procedure provides sufficiently good electrical and mechanical properties of wired contacts.

The quality of the contacts was tested by measuring a current - voltage characteristic of the wired fibers using Agilent E5270B precision measurement mainframe. Current flowing throw the fiber was limited to $30 \mathrm{~mA}$, since at higher current the nonlinearity of the current-voltage characteristic strongly increases and irreversible changes of the fibers electrical properties were detected. It seems that the overheating of the fiber is responsible for the irreversible changes of its electrical properties at higher current. From the linear part of the current - voltage characteristic the DC resistance of the sample $R$ was calculated. Resistivity of the sample was determined from the value of $R$ :

$\rho=R \frac{\pi D^{2}}{4 l}$;

where $D$ is a diameter of the fiber and $l$ is a distance between contacts. The value of $\rho$ was as a gauge of the quality of the contacts.

\subsection{Measurement results and discussion}

Measured current - voltage characteristics for A type samples are shown in Fig. 10. It can be seen that in the small voltage limit the current - voltage characteristic becomes linear whereas it turns into superlinear at higher voltage. Superlinearity might be caused by the heating of the fiber since the resistance of the ceramic samples decreases when the temperature increases. Calculated values of the specific resistivity are presented in the figure. It is seen that specific

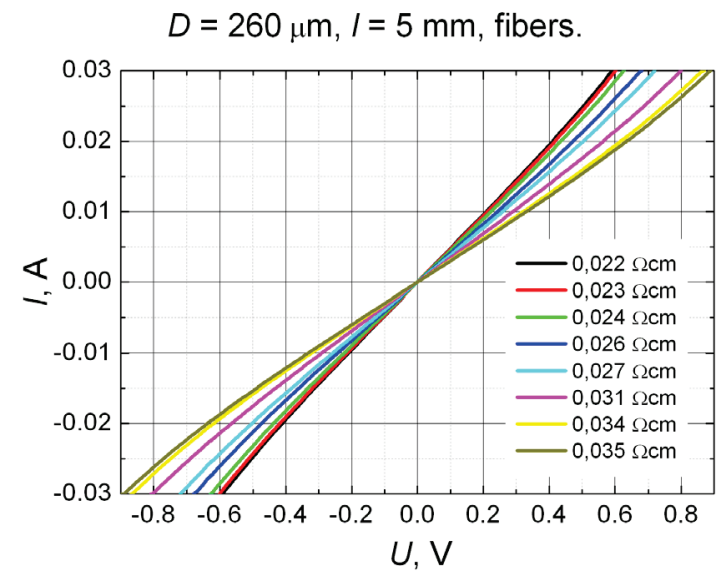

Fig. 10 Current-voltage characteristic of fibers with wires attached to the ends of the sample (Type A). 
Table 1 Averaged resistivity of investigated fibers

\begin{tabular}{ccc}
\hline $\begin{array}{c}\text { Contact } \\
\text { type/Number } \\
\text { of samples }\end{array}$ & $\begin{array}{c}\text { Average specific } \\
\text { resistance } \Omega \bullet \mathrm{cm}\end{array}$ & $\begin{array}{c}\text { Standard devi- } \\
\text { ation } \Omega \bullet \mathrm{cm}\end{array}$ \\
\hline A $/ 8$ & 0.028 & \pm 0.005 \\
B $/ 6$ & 0.026 & \pm 0.005 \\
C/11 & 0.029 & \pm 0.003 \\
\hline
\end{tabular}

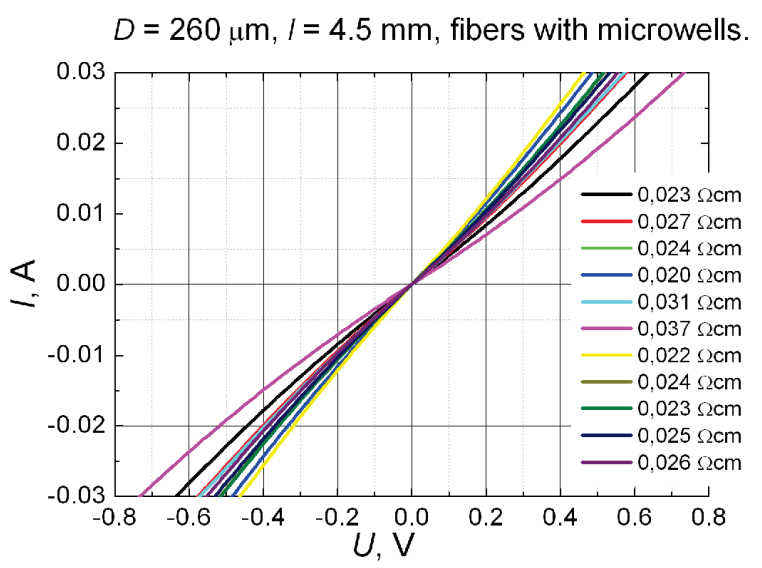

Fig. 11 Current-voltage characteristic of fibers with wires fixed in the microwells (Type B).

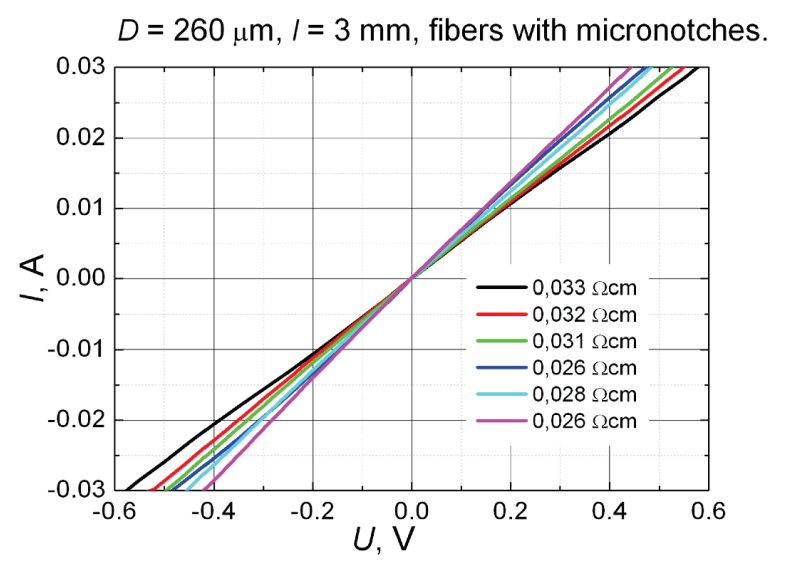

Fig. 12 Current-voltage characteristic of fibers with wires fixed in the micronotches (Type C).

resistivity of the samples falls in the range of $0.022 \Omega \mathrm{cm}$ $0.035 \Omega \mathrm{cm}$.

Current - voltage characteristics of micromachined samples - type B and C - are shown in Fig. 11 and 12, respectively. In general, they are similar to the characteristics shown in Fig. 10. Determined specific resistances are within intervals $0.020 \Omega \mathrm{cm}-0.037 \Omega \mathrm{cm}$ for samples with drilled wells and $0.026 \Omega \mathrm{cm}-0.033 \Omega \mathrm{cm}$ for samples, the wires for which are fixed in notches. Comparing results presented in Fig. 10 - 12, one can conclude that more sophisticated technology for the micromachining of cavities in the fibers does not have noticeable influence to their electrical conductivity. This conclusion is also confirmed by the results of averaged specific resistance shown in Ta- ble 1. In the first column of the table the type of the contact and number of tested samples is presented, and corresponding values of averaged specific resistance and their standard deviation are shown in the second and third columns of the table, respectively. Having in mind a standard deviation, one can conclude that there is no detectable difference in measured averaged specific resistance between reference (A) and micromachined (B and C) ceramic fibers.

In conclusion we can state that using femtosecond laser pulses it is possible to micromachine ceramics, sensitive to thermal stress. Material sustains all its conductive properties after micromachining.

\section{Acknowledgments}

This work was supported by research grant No. VP1-3.1SMM-10-V-02-007 (Development and utilization of a new generation industrial laser material processing using ultrashort pulse lasers for industrial applications) from the European Social Fund Agency. PR and ŽK thanks European Union Seventh Framework Program (FP7/20072013) under grant agreement No. CP-TP 229099-2 as part of the "MesMesh" project. Authors are grateful to G. Račiukaitis and B. Voisiat, (from FTMC, Lithuania) for additional micromachining of ceramic samples and D. Regonini, A. Dent and V. Adamaki (from BATH, UK) for ceramic sintering.

\section{References}

[1] R.R. Gattass, E. Mazur: Nat. Photonic, 2(4), (2008) 219.

[2] J. Kruger; W. Kautek: Laser Phys., 9(1), (1999) 30.

[3] G. Kamlage, T. Bauer, A. Ostendorf, B.N. Chichkov, Appl. Phys. A, 77(2), (2003) 307.

[4] X. Liu; D. Du; G. Mourou: IEEE J. Quantum Electron., 33(10), (1997) 1706.

[5] C.Y. Chien, M.C. Gupta: Appl. Phys. A, 81(6), (2005) 1257.

[6] Project "MesMesh" (Ultra - thin conductive ceramic mesh to monitor stress and wear on a steel surface) funded by the European Commission under the FP7 Cooperation Program: http://mesmesh.eu/ (checked 16/07/13).

[7] J. Ihlemann, A. Scholl, H.Schmidt, B. Wolffrottke: Appl. Phys. A, 60(4), (1995) 411.

[8] D. Regonini, A.C.E. Dent, C.R. Bowen, S.R. Pennock and J. Taylor: Mater. Lett., 65, (2011) 3590.

[9] A. Baškevičius, A. Alesenkov, G. Chaževskis, J. Litvaitytè, O. Balachninaitè, D. Paipulas, A. Melninkaitis and V. Sirutkaitis: J. Laser Micro Nanoeng., 8(1), (2013), 24.

(Received: July 19, 2013, Accepted: October 29, 2013) 